\title{
COUROUPITA GUIANENSIS LEAF CALLUS EXTRACT MEDIATED SYNTHESIS AND ANTIBACTERIAL ACTIVITY OF SILVER NANOPARTICLES
}

\author{
VENKATA RAJESH KUMAR T ${ }^{1}$, NARAYANA RAO MADAMSETTII ${ }^{1,2}$, BHARGAVA $\mathbf{Y}^{1,2}$, MURTHY JSR ${ }^{1 *}$
}

${ }^{1}$ Department of Botany, S. V. University, Tirupati, Andhra Pradesh, India. ${ }^{2}$ Department of Biotechnology, S. V. University, Tirupati, Andhra Pradesh, India. Email: jsrmurty@gmail.com

Received: 12 January 2016, Revised and Accepted: 09 February 2017

\section{ABSTRACT}

Objectives: Synthesis and evaluation of antimicrobial potential of silver nanoparticles (AgNPs) using aqueous leaf callus extract of Couroupita guianensis.

Methods: AgNPs were fabricated by green synthesis method using an aqueous extract of callus as agent of reduction and stabilization. AgNPs formation and their elemental nature were confirmed by ultraviolet (UV)-absorption spectroscopy and energy dispersive X-ray spectroscopy, respectively. Morphology of AgNPs was characterized by scanning electron microscope and atomic force microscope. Functional groups of biomolecules associated with AgNPs were inferred from characteristic Fourier transform infrared spectroscopy (FTIR) peaks. Antimicrobial activity of the AgNPs was assessed on MTCC strains by Kirby-Bauer disc diffusion method.

Result: Synthesized AgNPs exhibited characteristic UV/VIS absorption spectrum peaks at $410 \mathrm{~nm}$. The sizes of metallic spherical nanoparticles ranged from $30.38 \mathrm{~nm}$ to $88.32 \mathrm{~nm}$ and were in small aggregates. FTIR spectroscopy peaks of AgNPs, 3268, 1603, 1395, 1319, and $1045 \mathrm{~nm}$ corresponds to carbons of hydroxyl, amines, and ethers groups. Antimicrobial activity of AgNPs against tested Gram-positive and negative bacterial strains is significant compared to silver ions and gentamicin, and the zone of inhibition ranged from $16 \pm 0.8 \mathrm{~mm}$ to $20 \pm 0.1 \mathrm{~mm}$.

Conclusions: Stable AgNPs were synthesized through reduction and capping of silver ions by polyphenols and proteins present in callus extract. The enhanced antimicrobial activity of AgNPs may due to their small size leading to efficient molecular contact with the cell surface, penetration, and interaction, and inactivation of vital biomolecules.

Keywords: Couroupita guianensis, Callus extract, Silver nanoparticles, Antibacterial activity.

(c) 2017 The Authors. Published by Innovare Academic Sciences Pvt Ltd. This is an open access article under the CC BY license (http://creativecommons. org/licenses/by/4. 0/) DOI: http://dx.doi.org/10.22159/ajpcr.2017.v10i5.17066

\section{INTRODUCTION}

Biofabrication of silver nanoparticles (AgNPs) using live plants and extracts of plant tissues have received significant attention because of simple, cost-effective, and eco-friendly nature of the process compared to toxic chemical and physical methods. Many biomolecules generally found in plants such as proteins/enzymes, amino acids, polysaccharides, alkaloids, alcoholic compounds, polyphenols, and vitamins are attributed to bioreduction, formation, and stabilization of metal nanoparticles [1]. The nanoparticle synthetic potential, their size, and morphology depend on composition and concentration of biomolecules involved in the reduction of metal ions and stabilization of so-formed nanoparticles. In natural ecosystems metabolite composition is dynamic and changes with plant species dependent and environmental factors, accordingly nanoparticle synthetic potential also vary [2]. Hence, identification of plant-based system, which is a source of stable metabolite composition and capable of generating metallic nanoparticles reproducibly is essential.

The in vitro culture of cells and organs has gained prominence for the controlled production of plant natural products, due to the inherent advantages compared to naturally growing plant systems. Among the in vitro systems, callus cultures form the first step and basis for the establishment of cell cultures for the production of secondary metabolites. Several major classes of important bioactive constituents of naturally growing plants, viz., phenolic compounds, alkaloids, tannins, terpenoids, saponins, quinones, flavonoids, steroids, cardiac glycosides, and oils were reported from callus cultures, and in some cases, novel compounds were recorded. Callus cultures have a higher rate of metabolism than field grown plants due to nutrient-rich medium, and suitable controlled environmental conditions lead to the rapid proliferation and biosynthesis of active metabolites [3]. This proliferation and metabolic activity can be maintained by regular transfer of portions of callus into fresh medium. Hence, callus cultures are immortal and can ultimately provide a continuous, reliable source of bioactive natural products, which can be used for fabrication of metallic nanoparticles. The potential of calli from different plants in the synthesis of metallic nanoparticles, with novel shapes and varied sizes needs through investigation. In recent years, there are very few reports on the synthesis of AgNPs using callus cultures, and their application as antimicrobials and anticancer agents [4]

Couroupita guianensis, the most commonly known as Cannon ball tree, listed in threatened plant list and found rarely in Botanical gardens and temples in India [5]. Couroupita plant parts (leaves, flowers, fruit, and bark) possess antibacterial, antifungal, antiseptic, and analgesic qualities [6]. Phytochemical constituents with novel structures and bio-active moieties viz. stigmasterol, isatin, indirubin, eugenol and linalool, and alkaloids, phenolics and flavonoids etc. have been reported from different parts of this plant $[7,8]$. High amount of quercetin and stigmasterol from flowers was also reported by high performance thin layer chromatography method [9]. Many of the above phytochemical species reported from the various parts of this plant implicated in antimicrobial activity, and reduction of silver ions and stabilization of AgNPs in other plant systems.

Earlier we have reported the efficient synthesis of AgNPs using extracts of young flower buds and demonstrated their significant antibacterial activity [1], which indicates the genetic potential of synthesis of metabolites capable of nanoparticle fabrication. As in vitro callus 
cultures are metabolically highly active and a constant source of metabolites, this investigation was aimed at evaluation of nanoparticle synthetic potential of leaf callus extract and their antibacterial activity for the first time.

\section{METHODS}

Establishment of callus cultures and preparation of extract Callus cultures were established using explants from fully expanded young leaves collected from $C$. guianensis tree from the botanical garden of Sri Venkateswara University, Tirupati. Murashige and Skoog basal medium supplemented with 2,4-dichlorophenoxyacetic acid $1.0 \mathrm{mg}^{-1}$ and sucrose $3 \%$ was used for callus induction and subsequent subcultures [10] as shown in (Fig. 1). Aqueous extract of callus was prepared using actively proliferating callus from the first subculture. About $10 \mathrm{~g}$ of fragile callus were ground in $100 \mathrm{ml}$ of warm $35 \pm 2^{\circ} \mathrm{C}$ sterile distilled water and filtered through Whatman No. 1 filter paper and stored at $4^{\circ} \mathrm{C}$ in the refrigerator for further use.

\section{Synthesis of AgNPs}

After preliminary optimization, $1 \mathrm{ml}$ of callus extract was mixed with $30 \mathrm{ml}$ of silver nitrate $1 \mathrm{mM}$ solution without adjusting the $\mathrm{pH}$ and incubated at room temperature $28^{\circ} \mathrm{C}$ in dark for about $24 \mathrm{hrs}$. Initially, the progress of the reaction was monitored visually and later to confirm the reduction of silver ions the absorbance of the reaction mixture was measured after $1 \mathrm{hr}$ from 300 to $600 \mathrm{~nm}$ using UV-VIS spectrophotometer (UV-2600, Shimadzu Corporation, Kyoto, Japan) to find out the characteristic absorbance peak and stability of AgNPs.

\section{Characterization}

The stable AgNPs colloid was centrifuged at 10,000 g for 15 minutes, and the pellet was washed three times with double distilled water and dried in a freeze dryer to remove excess silver ions and used for characterization.

\section{Physical characterization}

Scanning electron microscope (SEM) and energy dispersive X-ray spectroscopy (EDAX)

The sample was prepared by dissolving the powder in milli-Q water; the solution was kept on a sonicator for 5 minutes. Then, a small droplet $20 \mu \mathrm{l}$ was kept on a small glass disc and dried at room temperature. SEM images were obtained using (CARL.ZEIS EVO MA 15 SEM). Elemental nature of silver nano powder was analyzed using (OXFORD INCA PENTA FET X3 EDS) instrument attached to SEM.

\section{Atomic force microscope (AFM)}

A droplet of purified AgNPs solution dried at room temperature on aluminum disc and subjected to AFM analysis through (NT-MDT SOLVER NEXT Germany) scanning probe microscope to know size, shape, and frequency of AgNPs.

\section{Chemical characterization}

\section{Fourier transform infrared spectroscopy (FTIR)}

FTIR spectrum of the dried fine powdered sample was recorded on Bruker, vertex K-ALPHA interferometer, Germany, in the range of $500-4000 \mathrm{~cm}^{-1}$ without adding the $\mathrm{KBr}$ pellets, to know the functional groups of stabilizer molecules.

\section{Antibacterial activity}

The antibacterial activity of AgNPs against pathogenic bacteria was examined using the standard Kirby-Bauer disc diffusion method. Bacterial strains, Bacillus cereus (MTCC - 4079), Micrococcus luteus (MTCC - 7256), Escherichia coli (MTCC - 1668), and Klebsiella pneumoniae (MTCC - 7028) were obtained from Microbial Type Collection Center, Chandigarh, India, and cultured in NB media at $37^{\circ} \mathrm{C}$. The overnight grown bacterial suspensions were swabbed on $4 \mathrm{~mm}$ thick MHA plates. Discs were prepared using $20 \mu \mathrm{L}$ each of the test solutions and gentamicin was used as a positive control and for comparison. Cultures were incubated at $37^{\circ} \mathrm{C}$ for $24 \mathrm{hrs}$, and the zone of inhibition was measured by MIC scale. For each treatment triplicates were performed. The results were subjected to one-way ANOVA.

\section{RESULTS AND DISCUSSION}

Synthesis of nanoparticles by callus extracts and cultures and their promising applications encouraged us to investigate AgNPs synthetic potential of $C$. guianensis callus extract and antibacterial activity of AgNPs. Colloidal AgNPs exhibit characteristic yellowish-brown color due to the interaction of electromagnetic radiation and electrons in the band around the nanoparticles. Initiation of bioreduction of silver ions by the aqueous extract of $C$. guianensis callus and progression of AgNPs synthesis was observed primarily by the change of color- less reaction mixer to yellowish brown. The color change of reaction mixture started almost instantaneously, the characteristic yellowish-brown color appeared rapidly within 2 minutes, and gradually the broth attained chocolate brown color within 15 minutes. The intensity of the yellowish-brown color of the solution increased with reaction time, and stable reddish-brown color appeared within 2 hrs and remained stable even after 1 month as shown in (Fig. 2). Rapid completion of reaction 2 hrs was also reported by Vigna radiata callus mediated synthesis, however, the ratio of callus extract to silver nitrate solution is very high 1:9 compare to this investigation 1:3000 [11]. Rapid synthesis of AgNPs in this investigation may be due to high concentration or efficient reducing agents present in the callus extract. AgNPs are known to exhibit size and shape-dependent surface plasmon resonance

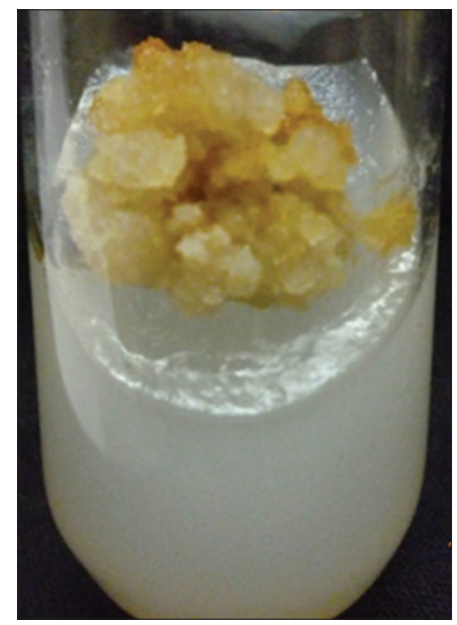

Fig. 1: Proliferating callus from leaf explants cultured on M.S. medium with 2,4.D

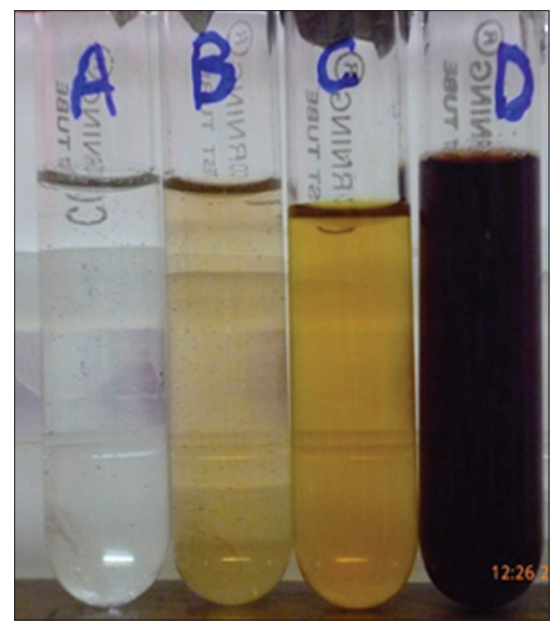

Fig. 2: Rapid color change of $\mathrm{AgNO}_{3}$; A (0-5) B (5-10) C (10-15) minutes D (2 hrs) 
(SPR) allowing simple monitoring of the formation of Ag nanoparticles reduction of $\mathrm{Ag}^{+}$ions to Ag0 by UV-VIS spectroscopy [12].

Here, AgNPs synthesis was monitored by UV spectra between 300 and $700 \mathrm{~nm}$ and confirmed by the characteristic SPR band at $410 \mathrm{~nm}$ as shown in (Fig. 3). The peak with an absorption maximum at $410 \mathrm{~nm}$ is narrow and single which indicates spherical nature of AgNPs [1]. The stability of AgNPs was confirmed by the stable reddish-brown color and SPR band at $410 \mathrm{~nm}$ even after 1 month storage in a refrigerator. This absorption maximum slightly deviates from the earlier callus mediated reports, which is due to the variation in reducing and capping agents [4]. A combination of highly efficient metal ions reducing and stabilizing agents present in the metabolically active callus is responsible for the synthesis of stable AgNPs. Compared to the young flower buds of C. guianensis [1] AgNPs were synthesized efficiently by the metabolites of callus. The efficient synthetic capability of callus compared to the differentiated tissue of intact plants was also reported $[11,13]$.

Efficient AgNPs formation was achieved with callus from the primary explants and synthetic potential of the callus extract slightly decreased with the number of subcultures. The efficiency of Sesuvium portulacastrum callus extract in a fabrication of distinct and uniformly scattered stable AgNPs compared to intact leaf extract was also demonstrated [13]. This may be due to insufficient or low active biomolecules present in terminally differentiated quiescent tissue of intact organs of the plants used for the synthesis of nanoparticles. The size and morphology of AgNPs generated by employing plant callus/ callus extract to some extent depend on metabolite composition and their concentration, which can be observed from microscopic images. SEM image of AgNPs indicating a high density of spherical particle varied in size, range from $30.38 \mathrm{~nm}$ to $88.32 \mathrm{~nm}$ and was in small aggregates as shown in (Fig. 4). This aggregation may be due to the high concentration of capping biomolecules controlling nanoparticle growth and clustering, as suggested earlier [12]. The presence of elemental Ag in the colloid was confirmed by the EDAX peak pattern in the silver region as shown in (Fig. 5).

The 3D-dimensional AFM image shows spherical and oval shapes, and colloidal nature of AgNPs, which is evident from (Fig. 6). The sizes of the nanoparticles are in agreement with SEM image and maximum frequency of nanoparticles is within the range of $40-50 \mathrm{~nm}$. The high frequency of spherical shaped AgNPs observed in AFM is in agreement with earlier reports [14]. Spherical shaped nanoparticles and size variation from few $\mathrm{nm}$ to tens of $\mathrm{nm}$ reported in many callus mediated fabrications [13]. The high density of spherical AgNPs was also reported from $V$. radiata [11]. The size variation is due to the variety of biomolecules with different molecular makeup associated with the particles. However, multiple shapes and $\mathrm{pH}$ dependent size variation were also reported earlier in callus extract mediated fabrications $[12,15]$. The possible reason for the variation in size and shape may due to the low level of expression of certain metabolic pathways resulted in a low concentration of metabolites involved in reduction and stabilization of AgNPs and not due to incomplete metabolic pathways in undifferentiated callus [12].

The $\mathrm{pH}$ dependent size variation observed by earlier investigators may be due to alteration of activity and interaction of biomolecules or broth with metal ions at different $\mathrm{pH}$. Biologically synthesized AgNPs are stabilized by a variety of biomolecules holding the metallic nanoparticles with their functional groups. Different possible nanoparticle stabilizers can be identified with their characteristic functional groups through FTIR analysis. The major photoelectron emissions peaks measured by FTIR spectroscopy are 3268, 1603, 1395,1319 , and $1045 \mathrm{~nm}$ correspond to carbons of hydroxyl, amines and ethers groups of amino acid residues of proteins, and polyphenols. A minor peak $1319 \mathrm{~nm}$ is representing Alkyl halide as shown in (Fig. 7). Similar FTIR peaks, 1603 and $1045 \mathrm{~nm}$ representing carbonyl and ether groups were also reported in Terminalia chebula extract stabilized SNPs [16]. In general, in rapidly proliferating cells, primary metabolic pathways are highly expressive and produce enzymes and proteins necessary for duplication of genomes and cell division. Moreover, in metabolically active cells antioxidative enzymes protect the integrity of the genome from the damage caused by highly reactive oxygen species generated in oxidative metabolism. The reducing agents are supposed to be members of antioxidants and the stabilizer could be belonging to polyphenols. Phenols are also considered as antioxidants and antibacterial compounds. Hence, we presume that possible reducers of silver ions and AgNPs stabilizer are mostly polyphenols and proteins, as inferred from functional groups represented by FTIR spectral peaks. Capping of AgNPs by proteins and polyphenols was also reported [17]. The present FTIR spectrum is similar to the stabilized AgNPs fabricated using the exudates of Medicago sativa callus [12].

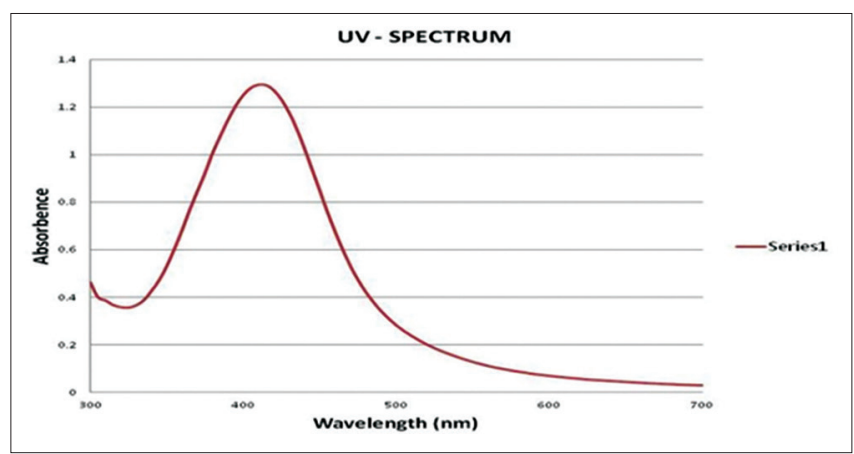

Fig. 3: Ultra violet-visible spectra of silver nanoparticles synthesized by leaf callus extract

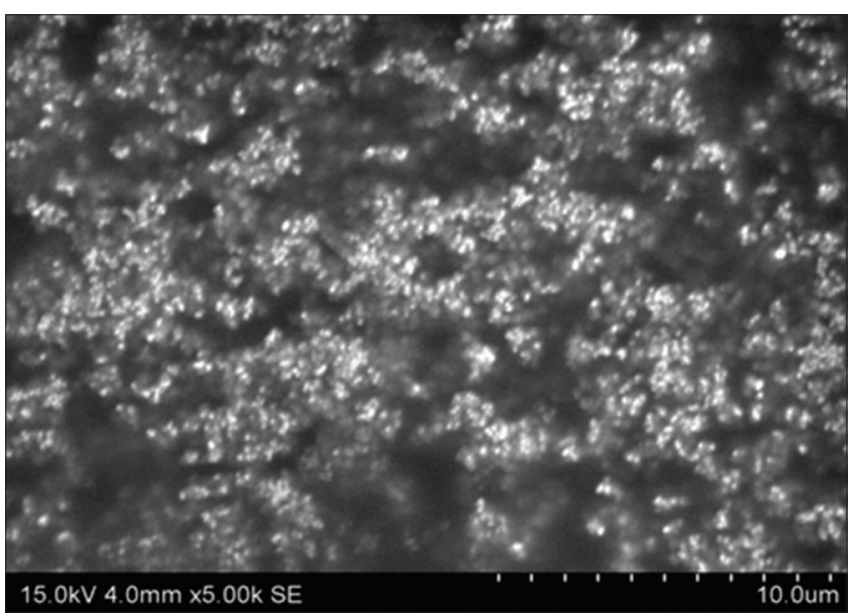

Fig. 4: Scanning electron microscope image of silver nanoparticles

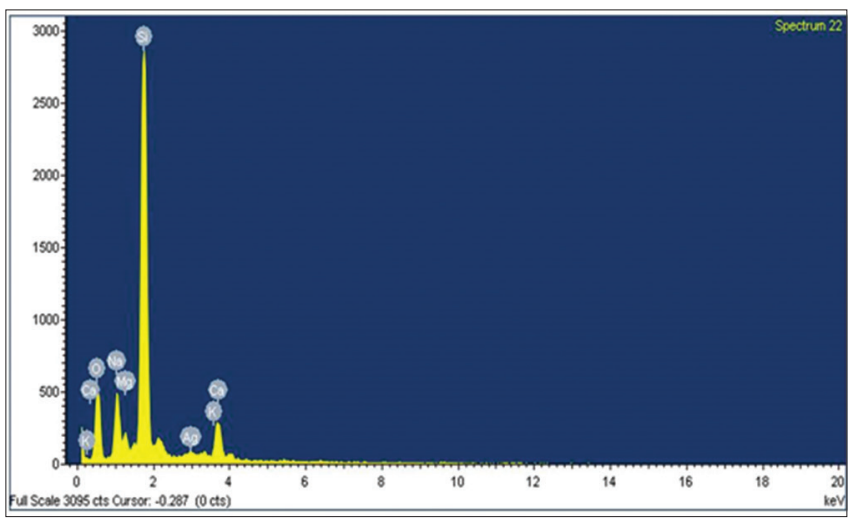

Fig. 5: EDS pattern of silver nanoparticles 
Antibacterial activity

Growing threat to public health posed by multidrug resistant bacteria emphasize the urgency to develop novel antimicrobial drugs with new multiple mechanisms of actions, which will control bacteria and emergence of resistance. Considering well known antibacterial activity of silver, research in medical and consumer products industry has attracted the use AgNPs as an antimicrobial agent in pharmaceutical preparations, dressings, food packing, and more recently in agriculture to control plant pathogens [18]. A wide range of important metabolites, flavonoids as well as phenolics with antimicrobial activity has been reported from C. guianensis. Further, C. guianensis flower buds extract mediated synthesis of AgNPs and enhanced antibacterial activity was also reported recently [1]. In this investigation, the antibacterial activity of AgNPs tested against two strains of each belongs to Gram-positive and negative groups. The antibacterial activity of AgNPs was observed in the form of a zone of inhibition and was compared with silver nitrate solution, aqueous extract of callus and standard drug, gentamicin which is shown in Fig. 8

Antibacterial activity of AgNPs against Gram-positive stains varied with the treatments. AgNPs displayed high antibacterial activity in the form of a maximum zone of inhibition, i.e., $17 \pm 0.2 \mathrm{~mm}$ and $16 \pm 0.8 \mathrm{~mm}$ in the case of B. cereus (MTCC - 4079) and M. luteus (MTCC - 7256), respectively (Table 1 ). This zone of inhibition is also significant, compared with gentamicin. In the case of Gram-negative strain, E. coli (MTCC - 1668) AgNPs were less inhibitory compared to gentamicin. However, the zone of inhibition by AgNPs significantly high compared to silver nitrate and callus extract. Further, AgNPs displayed maximum $20 \pm 0.1 \mathrm{~mm}$ zone of inhibition against K. pneumoniae (MTCC - 7028) which is significantly higher even compared to gentamicin $16 \pm 0.1$ (Table 1). The distinct antimicrobial activity of AgNPs fabricated by callus extracts against clinical bacterial strains was also reported previously [12].

When the zone of inhibition compared between Gram-positive and negative strains, AgNPs are equally effective against both groups. However, AgNPs fabricated by Taxus yunnanensis callus displayed significantly high inhibition against Gram-positive bacteria compared to Gram-negative bacteria [4]. This may be due to the difference in strains used and stabilizers associated with AgNPs. Among the four bacterial strains, K. pneumoniae (MTCC - 7028) which is Gram-negative is more sensitive to AgNPs. This may be due to the better interaction of AgNPs with Klebsiella cell membrane and their uptake making highly sensitive.

Several recent antimicrobial studies with biologically synthesized AgNPs established that nanoparticles efficiently interact with bacterial membranes and enter into the cell due to their nano size and high contact surface area to volume ratio. The nanoparticles interact with vital biomolecules (proteins/DNA) on the membrane and in cells leading to a range of effects from growth inhibition, loss of infectivity and cell death. AgNPs affect bacterial cell viability through the creation of pores on bacterial cell walls [19]. Extensive DNA fragmentation of human epidermoid larynx carcinoma (HEp-2) cell line leading to cell death by apoptosis by AgNPs synthesized from calli was also reported [14]. The polypeptides act as antimicrobials through the formation of ion channels in the microbial membranes. Thus, the enhanced antimicrobial activity shown by AgNPs derived from callus extracts in this investigation may also be due to their nano form and stabilizer phytochemicals, viz., phenols and proteins interacting with bacterial membrane and inhibiting macro molecules functions leading to antimicrobial activity.

\section{CONCLUSIONS}

Phenolic compounds and proteins present in callus extract reduced silver ions and stabilized in nanoparticle form. The size of spherical

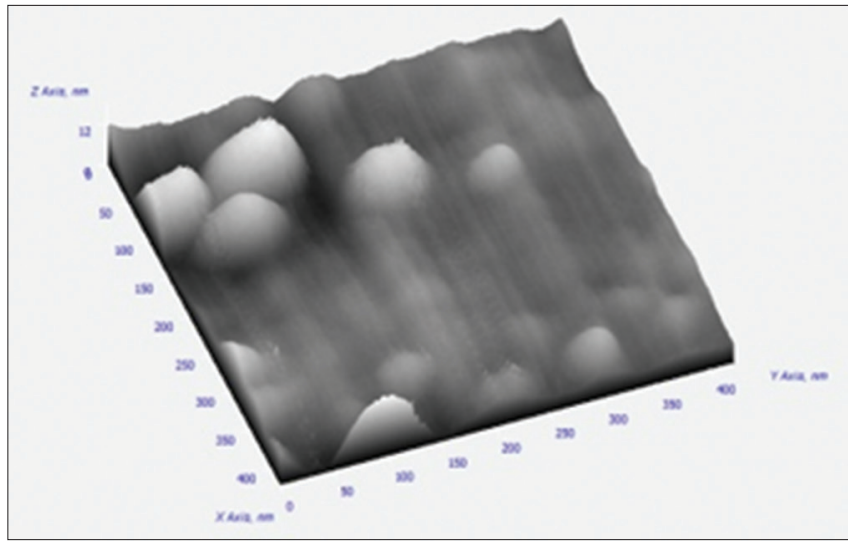

Fig. 6: Atomic force microscope image of silver nanoparticles

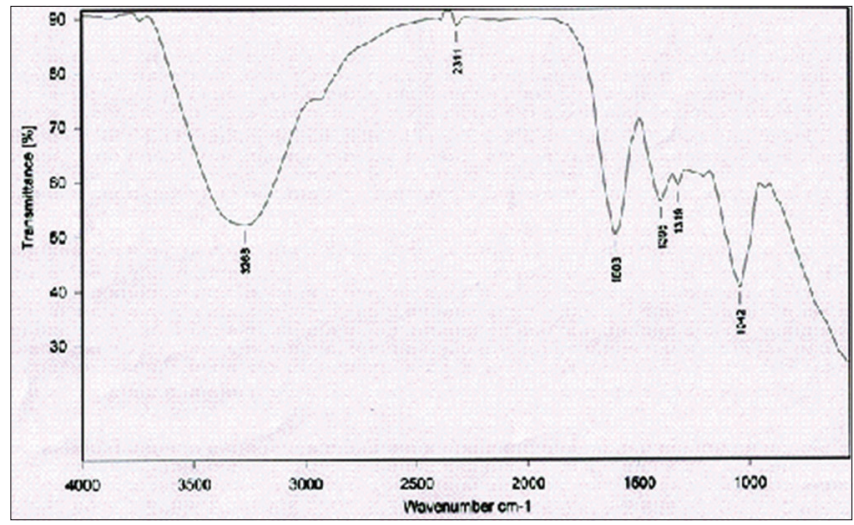

Fig. 7: Fourier transform infrared spectroscopy spectra of stabilized silver nanoparticles

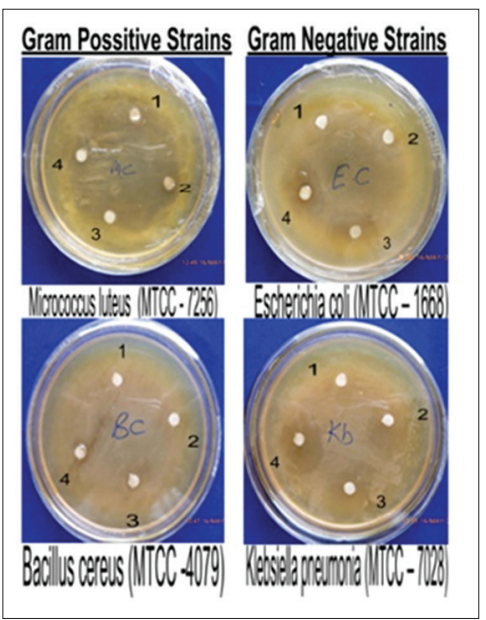

Fig. 8: Antibacterial activities of silver nanoparticles on Gram-positive and negative strains: (1) $\mathrm{AgNO}_{3}$, (2) AgNPs solution, (3) leaf callus extract, (4) gentamicin

nanoparticles ranged from 40 to $50 \mathrm{~nm}$. The AgNPs displayed significantly promising antimicrobial activity equally on tested Grampositive and negative bacteria compared to gentamicin.

\section{ACKNOWLEDGMENTS}

The first author Mr. T. Venkata Rajesh Kumar acknowledges the University Grants Commission, New Delhi, for providing financial assistance in the form of BSR-RFSMS fellowship. 
Table 1: Antibacterial activity of AgNPs on Gram-positive and Gram-negative strains

\begin{tabular}{llll}
\hline Bacterial strains & \multicolumn{2}{c}{ Zone of Inhibition (mm) } & \\
\cline { 2 - 4 } & AgNo $_{3}$ solution & Silver nano solution & Leaf extract \\
\hline Gram-positive, bacterial strains & & & Gentamicin \\
M. luteus (MTCC - 7256) & $12 \pm 0.1^{* *}$ & $16 \pm 0.8^{* *}$ & $10 \pm 0.4^{* *}$ \\
B. cereus (MTCC - 4079), & $11 \pm 0.7^{* *}$ & $17 \pm 0.2^{* *}$ & $09 \pm 0.2^{* *}$ \\
Gram-negative strains & $11 \pm 0.1^{* *}$ & $14 \pm 0.6$ & $12 \pm 0.1$ \\
E. coli (MTCC - 1668) & $15 \pm 0.7^{* *}$ & $14 \pm 0.6^{* *}$ & $08 \pm 0.5^{* *}$ \\
K. pneumonia (MTCC - 7028) & $20 \pm 0.1^{* *}$ & $13 \pm 0.2^{* *}$ \\
\hline
\end{tabular}

M. luteus: Micrococcus luteus, B. cereus: Bacillus cereus, E. coli: Escherichia coli, K. pneumonia: Klebsiella pneumonia, Significant after analysis of variance followed by Dennett's test. ${ }^{* *} \mathrm{p}<0.05$

\section{REFERENCES}

1. Kumar TV, Murthy JS, Rao MN, Bhargava Y. Evaluation of silver nanoparticles synthetic potential of Couroupita guianensis Aubl., Flower buds extract and their synergistic antibacterial activity. 3 Biotech 2016;92(6):1-9.

2. Moore BD, Andrew RL, Külheim C, Foley WJ. Explaining intraspecific diversity in plant secondary metabolites in an ecological context. New Phytol 2014;201(3):733-50.

3. Naik PM, Al-Khayri JM. Impact of abiotic elicitors on in vitro production of plant secondary metabolites: A review. J Adv Res Biotech 2016;1(2):1-7.

4. Xia QH, Ma YJ, Wang JW. Biosynthesis of silver nanoparticles using Taxus yunnanensis callus and their antibacterial activity and cytotoxicity in human cancer cells. Nanomaterials 2016;160(6):1-15.

5. Mitre M. Couroupita guianensis. In: IUCN Red List of Threatened Species, 2012. Version; 2012. Available from: http://www.iucnredlist.org.

6. Manimegalai S, Rakkimuthu G. Phytochemical screening of stem of Couroupita guianensis. Int J Pharm Sci Res 2012;3(11):4434-7.

7. Wong KC, Tie DY. Volatile constituents of Couroupita guianensis Aubl. Flowers. J Essent Oil Res 1995;7(2):225-7.

8. Rane JB, Vahanwala SJ, Goltkar SG, Ambaye RY, Khadse BG. Chemical examination of the flowers of Couroupita guianensis Aubl. Indian J Pharm Sci 2001;63:72-3.

9. Prabhu V, Subban R. Quantification of quercetin and stigmasterol of Couroupita guianensis Aubl by HPTLC method and in-vitro cytototoxic activity by MTT assay of the methanol extract against HeLa, NIH $3 \mathrm{t} 3$ and HEPG2 cancer cell lines. Int J Pharm Pharm Sci 2012;4(4):126-30.

10. Murashige T, Skoog F. A revised medium for rapid growth and bioassay with tobacco tissue cultures. Physiol Plant 1962;15(3):473-97.

11. Iyer RI, Selvaraju C, Santhiya ST. Biosynthesis of silver nanoparticles by callus cultures of Vigna radiata. Indian J Sci Technol 2016;9(9):1-5.

12. Lukman AI, Gong B, Marjo CE, Roessner U, Harris AT. Facile synthesis, stabilization, and anti-bacterial performance of discrete Ag nanoparticles using Medicago sativa seed exudates. J Colloid Interface Sci 2011;353(2):433-44

13. Nabikhan A, Kandasamy K, Raj A, Alikunhi NM. Synthesis of antimicrobial silver nanoparticles by callus and leaf extracts from saltmarsh plant, Sesuvium portulacastrum L. Colloids Surf B Biointerfaces 2010;79(2):488-93

14. Satyavani K, Gurudeeban S, Ramanathan T, Balasubramanian T. Biomedical potential of silver nanoparticles synthesized from calli cells of Citrullus colocynthis (L.) Schrad. J Nanobiotechnology 2011;9:43.

15. Hegazy HS, Rabie GH, Shaaban LD, Raie DS. Extracellular synthesis of silver nanoparticles by callus of Medicago sativa. Life Sci J 2014;11(10):1211-4.

16. Kirthika P, Dheeba B, Sivakumar R, Abdulla SS. Plant mediated synthesis and characterization of silver nanoparticles. Int $\mathrm{J}$ Pharm Pharm Sci 2014;6(8):304-10.

17. Mude N, Ingle A, Gade A, Rai M. Synthesis of silver nanoparticles using callus extract of Carica papaya-A first report. J Plant Biochem Biotechnol 2009;18(1):83-6.

18. Shah M, Fawcett D, Sharma S, Tripathy SK, Poinern GE. Green synthesis of metallic nanoparticles via biological entities. Materials 2015;8(11):7278-308.

19. Rai VR, Bai AJ. Nanoparticles and their potential application as antimicrobials. Science Against Microbial Pathogens, Communicating Current Research and Technological Advances. Vol. 1. Badajoz: Formatex; 2011. p. 197-09. 